\title{
Deux indices de compétition pour la comparaison de la croissance en hauteur et en diamètre d'arbres aux passés sylvicoles variés et inconnus
}

\author{
M Becker \\ INRA, Laboratoire de phytoécologie forestière, centre de recherches de Nancy, \\ 54280 Champenoux, France
}

(Reçu le 13 mai 1991; accepté le 4 octobre 1991)

\begin{abstract}
Résumé - Le travail présenté a pour l'instant un caractère essentiellement exploratoire. Les indices proposés reposent sur le "postulat" que le facteur d'élancement (H/D) d'un arbre dépend des conditions moyennes de compétition subies au cours de son développement mais est indépendant des conditions de station. II s'avère que la moyenne des facteurs d'élancement d'arbres soumis au même type de traitement sylvicole dépend aussi de l'âge, selon un modèle de la forme : Log $(H / D)=$ $a-b$-Age (1). Connaissant l'âge, la hauteur $H$ et le diamètre $D$ d'un arbre, on construit 2 indices correcteurs de compétition $C d$ et $C h$ tels que $C d . D=D r$ et $C h . H=H r, H r$ et $D r$ étant les dimensions d'un arbre de référence de même âge et de statut de compétition moyen. Les valeurs de $\mathrm{Hr}$ et $\mathrm{Dr}$ sont inconnues, mais le rapport $H r / D r$ peut être calculé grâce à la relation (1). Le rapport $C d / C h$ est donc connu, et appelé alpha. Le modèle très simple proposé actuellement pour parvenir aux indices de compétition est :
\end{abstract}

$$
C d=(\text { alpha })^{(1-k)} \text { et } C h=(a l p h a)^{-k}
$$

Les données dendrométriques disponibles sur 505 chênes pédonculés (Quercus robur $L$ ) du plateau lorrain ont permis de fixer empiriquement à 0,3 la valeur de $k$. Les premières applications pratiques des indices proposés ont utilisé les mêmes données, et celles de 529 chênes sessiles ( $Q$ petraea (Matt) Liebl) issus des mêmes forêts. Sans compensation de la compétition, la croissance radiale des 2 espèces ne présente pas de tendance significative à long terme, ce qui contraste avec les résultats obtenus précédemment sur le sapin (Abies alba Mill) et sur le hêtre (Fagus silvatica $L$ ) dans les Vosges et le Jura. Après compensation avec $C d$, on constate une nette dérive positive depuis $1830(+60 \%$ chez le chêne sessile, $+32 \%$ chez le chêne pédonculé). Par ailleurs, les taux de variance expliquée relatifs à la hauteur en fonction des variables stationnelles disponibles sont plus élevés avec utilisation de l'indice correcteur Ch que sans son utilisation (respectivement 29 et $22 \%$, et même 54 et $37 \%$ dans les milieux bien drainés). D'autres tests de validation méritent d'être faits, en particulier pour confirmer la solidité du postulat de base. En outre, le modèle (2) devrait pouvoir être amélioré. Les indices $C d$ et $C h$ pourraient être précieux pour l'étude des relations stationproduction dans des forêts au passé sylvicole perturbé et, en dendroécologie, pour celle du déterminisme écophysiologique de la croissance radiale des grandes essences forestières.

compétition / sylviculture / hauteur dominante / dendroécologie / croissance à long terme / relation station-production 
Summary - Two competition indices for comparing the height and the dlameter growth of trees characterized by various and unknown silvicultural histories. For the moment, the present study is mainly exploratory. The indices proposed are based on the "postulate" : the height/diameter ratio $(\mathrm{H} / \mathrm{D})$ of a tree depends on its average past competition status but is independent of the site conditions. The mean $(\mathrm{H} / \mathrm{D})$ ratio for trees subjected to the same silvicultural treatment is related to age too, in accordance with the following model: $\log (\mathrm{H} / \mathrm{D})=\mathrm{a}-\mathrm{b} \cdot \mathrm{Age}$ (1). The age, the height $\mathrm{H}$ and the diameter $\mathrm{D}$ of a given tree are measured. Then two compeition indices $\mathrm{Cd}$ and $\mathrm{Ch}$ are created as $\mathrm{Cd} \cdot \mathrm{D}=\mathrm{Dr}$ and $\mathrm{Ch} \cdot \mathrm{H}=\mathrm{Hr}$, where $\mathrm{Hr}$ and $\mathrm{Dr}$ are the dimensions of a reference tree that would be the same age and characterized by an average competition status. Both $\mathrm{Hr}$ and $\mathrm{Dr}$ are unknown, but the Hr/Dr ratio can be calculated according to (1). Thus, $\mathrm{Cd} / \mathrm{Ch}$ is well-defined, and called alpha. For the moment, a very simple model is proposed to produce the competition indices :

$$
\mathrm{Cd}=(\text { alpha) })^{(1-k)} \text { et } \mathrm{Ch}=(\text { alpha })^{-k}(2)
$$

Dendrometrical data available for 505 pedunculate oaks (Quercus robur $L$ ) from the Lorraine plateau were used to determine empirically $\mathrm{k}: \mathrm{k}=0.3$. Initial attempts have been achieved in order to test the relevance of the indices proposed, using the same data and those of 529 sessile oaks (Q petraea (Matt) Liebl) from the same forests. Without using the competition indices, the radial growth of both species does not exhibit any significant long term trend, which contrasts with previous results from Silver fir (Abies alba Mill) and Beech (Fagus silvatica $L$ ) in the Vosges and the Jura mountains. After compensating ring widths with $\mathrm{Cd}$, a clear increase since the year 1830 becomes evident: $+60 \%$ for sessile oak, $+32 \%$ for pedunculate oak. Furthermore, stepwise multiple regressions have been carried out in order to predict tree height using some ecological data. The variance explained is higher when the competition index $\mathrm{Ch}$ is used: $29 \%$ vs $22 \%$ without this use, and even $54 \%$ vs $37 \%$ in welldrained sites. Further validation tests are desirable, especially in order to confirm the reliability of the basic postulate. Moreover, it is likely that the model (2) could be improved. The indices $\mathrm{Cd}$ and $\mathrm{Ch}$ could be of great interest for studying site-yield relationships in the forests characterized by a perturbed silvicultural past, and, in dendroecological studies, for analysing the ecophysiological determinism of the radial growth of the main forest species.

competition / silviculture / site index / dendroecology / growth trend / site-yield relationships

\section{INTRODUCTION}

La définition d'indices dendrométriques fiables révélateurs de la productivité des types de stations est une préoccupation importante dans de nombreuses recherches forestières, tant appliquées, pour quantifier directement le potentiel de production de sites déterminés, que plus fondamentales, pour analyser le fonctionnement des écosystèmes forestiers à travers le déterminisme écophysiologique de la croissance.

Ces indices privilégient tantôt la croissance radiale, tantôt, et c'est le cas le plus fréquent, la croissance en hauteur.
La croissance radiale annuelle d'un arbre dépend pour l'essentiel des caractéristiques physico-chimiques de la station où il est installé, des conditions climatiques de l'année (voire des quelques années précédentes; Becker, 1989), mais aussi, pour une très large part, de son statut de compétition au sein du peuplement dont il fait partie. De plus, ce statut peut varier considérablement au cours de la vie de l'arbre, et l'on n'a qu'exceptionnellement la possibilité de reconstituer ce processus pour "expliquer" le diamètre observé à un instant déterminé. C'est pourquoi la croissance radiale n'est que rarement utilisée dans la construction d'indices de productivité (Day et al, 1960), et seulement dans le 
cas de plantations régulières et en l'absence d'éclaircies.

C'est à la croissance en hauteur qu'il est fait appel le plus souvent, en particulier à la hauteur dominante à un âge de référence donné (Décourt, 1973). Si I'on dispose d'une table de production valide dans la région et pour l'essence considérées, cet indice permet ensuite de passer à la production totale en volume à ce même âge. C'est par l'intermédiaire des hauteurs dominantes que nombre d'études de liaison station-production ont été réalisées à ce jour (Oswald, 1969; Décourt et Le Tacon, 1970; Le Tacon et Millier, 1970; Becker et al, 1980).

Là encore, le critère "hauteur dominante à un âge de référence" n'est cependant pleinement utilisable que pour comparer des peuplements ayant été soumis à une même sylviculture. En particulier, pour prendre un exemple caricatural, il est impossible, directement, de comparer les performances atteintes par 2 peuplements de chêne, l'un traité en futaie, l'autre en taillissous-futaie. Pour un type de station donné, donc une productivité déterminée, l'écart de hauteur dominante à 120 ans atteint plusieurs mètres, et peut être supérieur aux différences observées entre les meilleures et les moins bonnes stations. II en est de même pour le hêtre (Becker, 1978).

II serait donc de la plus grande utilité de pouvoir disposer de méthodes permettant de compenser les hauteurs ou les diamètres bruts mesurés en fonction du passé sylvicole des arbres, et ainsi d'aboutir à de nouveaux indices de productivité qui ne dépendent plus que des caractéristiques stationnelles. Des tentatives ont déjà eu lieu dans ce sens, en particulier celle de Le Goff (1984), sur la base de couples de placettes futaie/taillis-sousfutaie, pour étudier la productivité du chêne dans la région centre. lci, les conditions de concurrence moyennes subies par les arbres au long de leur vie ont été appréhendées à travers la morphologie du houppier, et plus précisément par la mesure du rapport de la hauteur du houppier à la hauteur totale de l'arbre. Ceci permet ensuite de prévoir de façon satisfaisante la productivité potentielle "futaie" de stations portant aujourd'hui des taillis-sous-futaie "candidats" à la conversion en futaie régulière.

Intuitivement, on peut cependant regretter que le paramètre dendrométrique de base soit la circonférence, et non la hauteur, dont on a vu plus haut qu'elle était un révélateur plus solide de la potentialité d'une station. Par ailleurs, sur un plan pratique, la mesure de la hauteur du houppier peut comporter une part de subjectivité assez critique selon l'architecture des arbres étudiés, qui est souvent plus ou moins déséquilibrée (houppiers dissymétriques, basses branches mortes, ou dressées, ou retombantes, présence de "gourmands" bas parfois très développés et représentant une part non négligeable de la masse foliaire, etc). L'approche alternative que nous proposons repose sur l'utilisation du ufacteur d'élancement" $(H / D)$, rapport de la hauteur totale de l'arbre à son diamètre à $1,30 \mathrm{~m}$, qui sont 2 grandeurs mesurables sans aucune ambiguiité.

La réflexion que nous avons développée est née de nos interrogations à l'occasion d'une étude dendroécologique en cours dans diverses chênaies du plateau lorrain. En effet, la plupart des études de ce type menées à ce jour dans les montagnes de l'Est de la France (Vosges et Jura), selon des démarches méthodologiques semblables, ont mis en évidence une très nette dérive positive de la croissance radiale depuis le début $\mathrm{du} X \mathrm{X} \mathrm{X}^{\ominus}$ siècle, tant chez le sapin pectiné - Abies alba Mill (Becker, 1989; Bert et Becker, 1990) que chez le hêtre - Fagus sylvatica - (Picard, 
comm pers). Chez le sapin, cette dérive a pu être clairement reliée aux facteurs climatiques, essentiellement la pluviométrie (Becker, 1989). Au contraire, dans un premier temps, celle du chêne, tant le chêne sessile - Quercus petraea (Matt) Liebl que le chêne pédonculé $-Q$ robur $L$-, ne semblait présenter aucune tendance significative à long terme (Nieminen, 1988). Plusieurs hypothèses pouvaient être avancées pour expliquer une telle différence de comportement :

- expression de différences spécifiques : le chêne aurait un comportement écophysiologique, sous déterminisme génétique, fondamentalement différent de celui des autres espèces, en particulier en ce qui concerne leur réaction aux facteurs climatiques; cette hypothèse ne pouvait être totalement écartée a priori mais ne paraissait guère convaincante;

- évolution à long terme des conditions climatiques différente sur le plateau lorrain et dans le massif des Vosges; ceci ne paraissait également que peu vraisemblable, mais ne pouvait être totalement exclu;

- expression des différences bioclimatiques des 2 étages de végétation prospectés (étage collinéen pour le chêne, étage montagnard pour les 2 autres espèces), où la hiérarchie des facteurs limitants de la croissance est elle-même probablement différente (pluviométrie dans le premier cas, température dans le second);

- conséquence des modifications profondes du traitement sylvicole appliqué aux forêts de chêne étudiées au cours du siècle passé, en particulier des efforts de conversion des anciens taillis-sous-futaie en futaie régulière; de telles opérations ont eu des répercussions évidentes sur la croissance des arbres disponibles aujourd'hui, qui pourraient avoir compensé la tendance générale observée ailleurs.

C'est pour éprouver cette dernière hypothèse que nous avons développé les travaux rapportés ici, qui ont surtout un caractère exploratoire. Ils reposent sur une approche à la fois théorique et empirique, que nous avons ensuite cherché à éprouver concrètement sur l'exemple des chênaies évoqué ci-dessus.

\section{MÉTHODE}

Le rapport hauteur/diamètre (H/D) est utilisé tantôt à l'échelle du peuplement - on l'appelle alors parfois "facteur de stabilité" - pour quantifier les risques de chablis importants (Oswald, 1984), tantôt pour des arbres individuels. Dans le second cas - on l'appelle alors plutôt "facteur d'élancement" - il ne s'agit pas seulement d'un coefficient de forme. II renseigne également sur la position sociale des arbres (Pardé et Bouchon, 1988) : «Les arbres dominants et codominants ont normalement un rapport inférieur à 100; et pour le choix des arbres d'avenir, on conseille de ne retenir que des arbres ayant un rapport inférieur à 80 \%.

Le rapport $(H / D)$ d'un arbre apparaît être un bon intégrateur des conditions moyennes de compétition auxquelles cet arbre a été soumis tout au long de sa vie; ces conditions de compétition englobent à la fois le statut social de l'arbre au sein du peuplement ef la densité de ce dernier. Le facteur d'élancement a récemment été mis à profit pour montrer la part importante de responsabilité d'une densité excessive ancienne (plus de 30 ans) dans le dépérissement actuel irréversible de diverses sapinières vosgiennes (Lévy et Becker, 1987).

Le "postulat" largement intuitif, sur lequel repose essentiellement notre argumentation, est le suivant : le facteur d'élancement $(H / D)$ d'un arbre résulte de la compétition moyenne subie depuis sa naissance mais est indépendant du type de station (au sens phytoécologique du terme; Delpech et al, 1985) sur lequel il a poussé. A ce stade, le postulat ci-dessus apparaît compatible avec les observations anciennes qui ont servi à fonder la "loi de Eichhorn élargie" (Gehrhardt, 1909; Assmann, 1955; Pardé et Bouchon, 1988), dont l'une des acceptions dit que le diamètre moyen d'un peuplement ne dépend que de sa hauteur moyenne, pour une sylviculture donnée, indépendamment du type de station et de l'âge du peuplement. 


\section{Recherche d'un estimateur de (H/D)}

Les données dendrométriques utilisées ont été recueillies sur 505 chênes pédonculés dans les forêts domaniales d'Amance et de Champenoux (54). Ces arbres ont été choisis parmi les dominants et codominants exclusivement, dans 115 placettes ( 1 à 5 arbres par placette) réparties de façon à représenter au mieux la variabilité des classes d'âge et des conditions stationnelles. En revanche, ils ne sont pas censés être représentatifs de la totalité des arbres des peuplements dont ils sont issus. Dans ces conditions, il s'avère que leur facteur d'élancement $(H / D)$ dépend aussi de leur âge (fig 1). Pour des raisons techniques, l'áge indiqué est l'âge à $2,80 \mathrm{~m}$, compté sur des carottes prélevées à cette hau-. teur. II est compris entre 10 et 332 ans (moyenne : 80 ans; écart type : 46 ans).

La figure 1 comporte à la fois des arbres issus de la conversion en futaie entreprise à partir de 1850 environ $(n=368)$, et des arbres dits de "taillis-sous-futaie" (TSF) ( $n=137)$, qui en conservent encore l'architecture au niveau des ramifications principales, mais qui sont en fait dans une phase de vieillissement plus ou moins avancée, préparatoire à la régénération. II est important de souligner ici que, d'une part, la variabilité des types de stations des 2 forêts est assez faible et d'autre part, la répartition des parcelles déjà en futaie et de celles en vieillissement est sensiblement indépendante de ces types stationnels.

Le modèle choisi pour rendre compte de la relation moyenne liant $(H / D)$ à l'âge est de la forme :

$$
\log (H / D)=a-b \cdot A g e(1)
$$

Les coefficients $a$ et $b$ ont été estimés, dans le cas rapporté ici, en ne prenant en compte que les seuls arbres de futaie. En effet, il est clair que les gammes d'åges disponibles en futaie et en TSF sont très différentes (respectivement $10-130$ ans et $70-330$ ans), ce qui aurait biaisé un ajustement indifférencié. On a alors :

$$
\begin{gathered}
a=4,708 ; \\
b=5,01.10^{-3} \\
\text { (écart type }=2,54 \cdot 10^{-4} \text { ) }
\end{gathered}
$$

écart type initial de $\log (H / D)=0,220$; écart type résiduel de $\log (H / D)=0,153$.

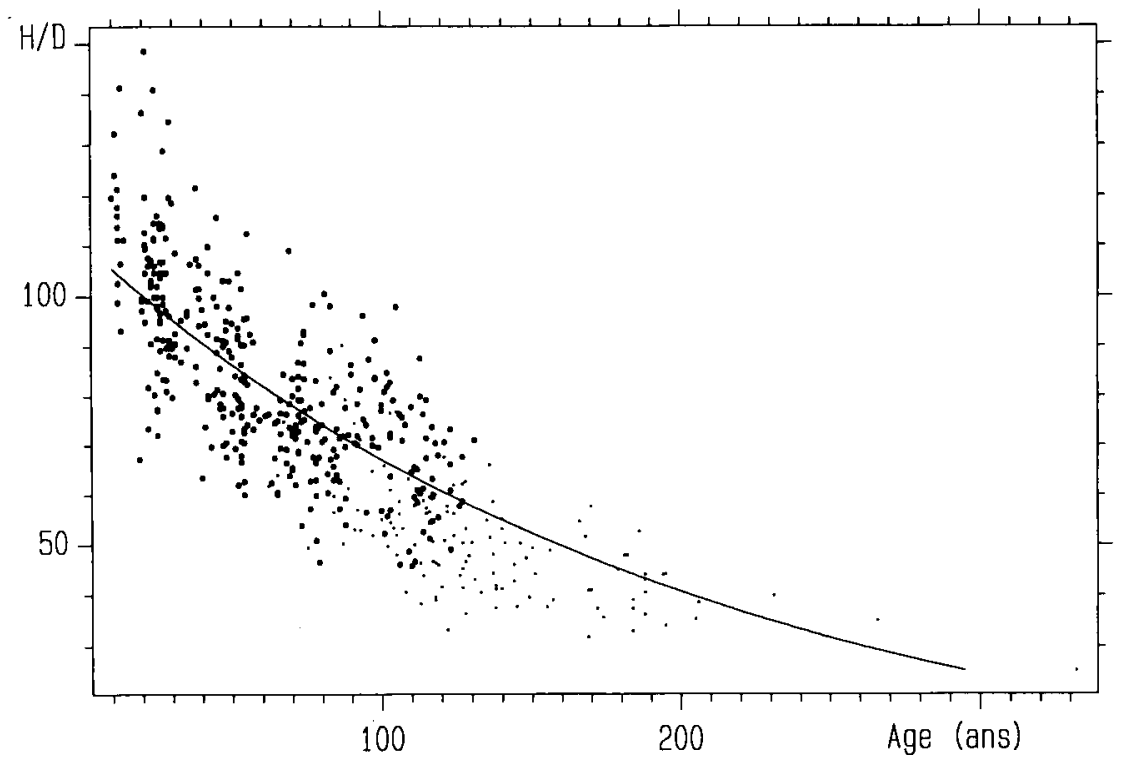

Fig 1. Rapport hauteur/diamètre $(H / D)$ en fonction de l'âge à $2,80 \mathrm{~m}$ pour le chêne pédonculé en forêts d'Amance et de Champenoux (54); gros points : 368 arbres de futaie; petits points : 137 arbres de taillis-sous-futaie. L'ajustement prend en compte les seuls arbres de futaie. 
Le taux de variance expliquée par le modèle ainsi obtenu est de $51,5 \%$. Son extrapolation jusqu'à 330 ans est, bien sûr, quelque peu critiquable. Mais d'une part, il n'y a guère d'alternative compte tenu de la structure des données disponibles et d'autre part, la courbe obtenue au-delà de 130 ans (fig 1) apparaît tout à fait cohérente avec la position relative des arbres de futaie et de TSF entre 70 et 130 ans (futaie : 148 arbres, $(H / D)$ moyen $=70,9$; TSF : 79 arbres, $(H / D)$ moyen $=56,1$; différence significative à $P<0,001$ ). La grande majorité des points TSF sont situés sous l'ajustement, tant avant qu'après 130 ans, ce qui est parfaitement logique et traduit le caractère plus trapu $[[H / D]$ plus faible) des arbres correspondants.

\section{Recherche d'indices moyens correcteurs des effets de la competition}

Pour un arbre déterminé, on dispose donc de 3 données : sa hauteur $H$; son diamètre $D$; un estimateur de son rapport $(H / D)$ déduit de son âge, appelé $f(A g e)$, et calculé à l'aide la formule (1).

On peut exprimer $f($ Age $)$ comme étant le rapport $H / D=H_{r} / D_{r}$ d'un arbre de référence de même âge, ayant été conduit en futaie, dans des conditions moyennes de compétition. Pour un type de station déterminé, on peut aisément admettre que les valeurs $H_{r}$ et $D_{r}$ sont uniques, mais, à ce stade, elles nous sont inconnues.

Le but est précisément de définir des coefficients $C d$ et $C h$ tels que :

$$
C d \cdot D=D_{r} \text { et } C h \cdot H=H_{r}
$$

Par définition, on a :

$$
C d / C h=(H / D) /\left(H_{r} / D_{r}\right)=(H / D) / f(\text { Age })=\text { alpha }
$$

La valeur alpha peut donc être calculée sans ambiguîté. Il s'agit en fait du facteur d'élancement $(H / D)$ standardisé en fonction de l'âge et, étant donné le choix des arbres fait pour l'estimation des paramètres du modèle (1), rapporté à un traitement en futaie menée dans des conditions moyennes de densité. Lorsque alpha est supérieur à 1 , on a affaire à des arbres de futaie ayant subi une compétition supérieure à la moyenne. Lorsque alpha est inférieur à 1 , il s'agit d'arbres ayant profité d'une compétition inférieure à la moyenne; la majorité des arbres de TSF sont dans ce cas.

Par définition, le rapport des indices $C d$ et $C h$, pour un arbre donné, a une valeur déterminée. Préciser la valeur respective de chacun d'eux n'est pas évident a priori. Le modèle suivant très simple peut être proposé, sans préjuger d'améliorations possibles ultérieures :

$$
C d=(\text { alpha })^{(1-k)} \text { et } C h=(a l p h a)^{-k}(3)
$$

II reste à déterminer la valeur du coefficient $k$, lequel traduit en fait la façon dont les effets de la compétition se répartissent entre la croissance en diamètre et la croissance en hauteur. Faute de basses théoriques pour y parvenir, nous avons opté pour une approche empirique, sur la base des données dendrométriques disponibles sur les 505 chênes pédonculés des forêts d'Amance et de Champenoux.

\section{Détermination du coefficient $\mathrm{k}$}

Calculée à l'aide de la formule (2), la valeur de alpha varie approximativement, dans les forêts étudiées, entre 0,5 (arbres les moins concurrencés) et 1,6 (arbres les plus concurrencés).

Il y correspond, tous traitements confondus, chez les arbres ayant atteint l'âge adulte et pour lesquels futaie et TSF sont bien représentés (110-130 ans) :

- des circonférences observées $C$ variant entre $251 \mathrm{~cm}$ et $106 \mathrm{~cm}$ (fig 2), soit dans le rapport $251 / 106=2,37$;

- et des hauteurs observées $H$ variant entre 20 et $32 \mathrm{~m}$ (fig 3), soit dans le rapport 20/32 =0,63.

Cette large amplitude de $C$ et de $H$ résulte a la fois des conditions locales moyennes de compétition - c'est la part dont on souhaite s'abstraire - et de la fertilité de la station - c'est celle que l'on souhaite extraire pour mieux pouvoir ensuite l'analyser et l'expliquer.

On peut calculer, pour une série de valeurs possibles de $k$ (de 0,1 à 0,5 ), les valeurs extrêmes de $C d\left(C d_{\min }\right.$ et $\left.C d_{\max }\right)$ et de $C h\left(C h_{\max }\right.$ et $C h_{\min }$ ) qui en résulteraient dans les 2 forêts étudiées, compte tenu des valeurs extrêmes 


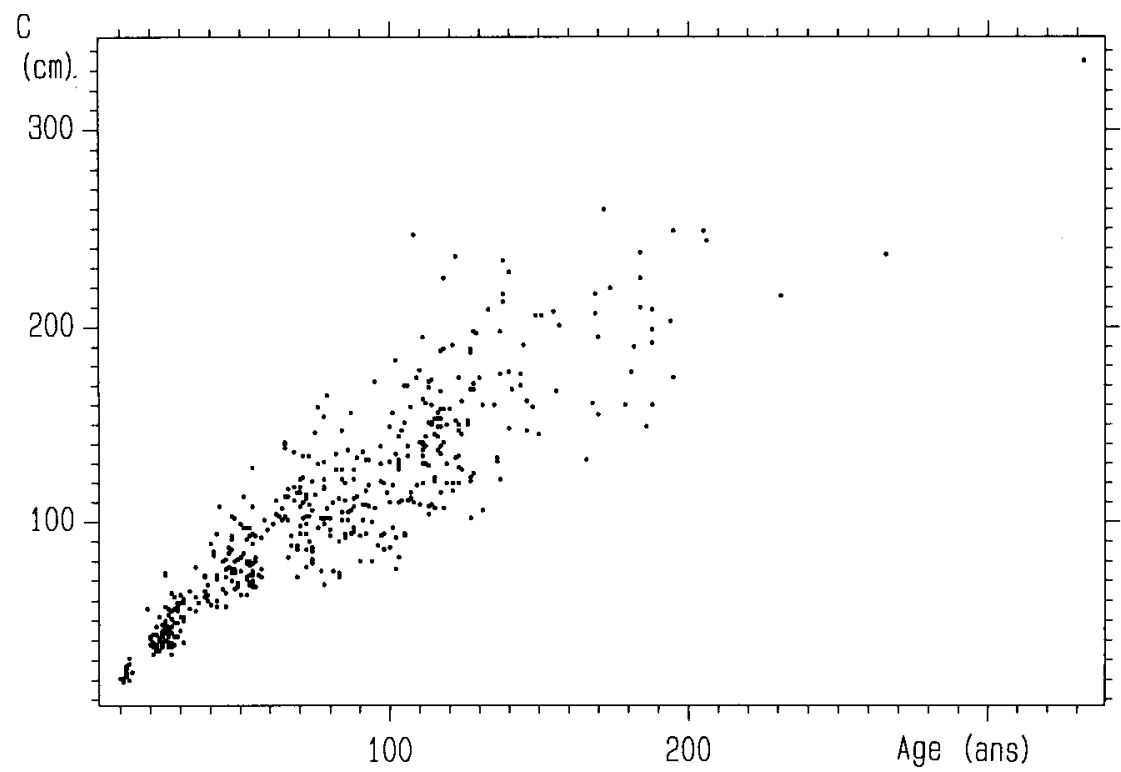

Fig 2. Circonférence $C$ en fonction de l'âge à $2,80 \mathrm{~m}$ pour 505 chênes pédonculés des forêts d'Amance et de Champenoux (54).

observées pour alpha $(0,5$ et 1,6 ; voir cidessus), ainsi que les rapports de ces extrêmes (tableau I).

On compare enfin les rapports 2,37 et 0,63 obtenus précédemment avec les rapports $C d_{\max } / C d_{\min }$ et $C h_{\min } / C h_{\max }$ ci-dessus. Le bienfondé de cette comparaison n'est, bien sûr, pas rigoureux, car les premiers traduisent l'effet cumulé de la compétition et de la station, alors que les seconds ne reflètent en principe que la seule compétition. Mais elle ne nous semble pas fondamentalement biaisée. En effet :
- d'une part, dans la gamme d'âges de référence choisie (110-130 ans), la fourchette des valeurs alpha observées tient en fait pour beaucoup au type de traitement (voir fig 1; futaie : 37 arbres, $(H / D)$ moyen $=64,2$; TSF : 46 arbres, $(H / D)$ moyen $=51,9$; différence significative à $P<0,001$ );

- d'autre part, comme il a déjà été signalé, la répartition relative des parcelles à structure de futaie et de celles à structure encore plus ou moins proche du TSF est sensiblement indépendante de celle des stations.

Tableau I. Valeurs extrêmes calculées de $C d$ et de $C h$ et rapport de ces extrêmes pour diverses valeurs envisageables du coefficient $k$ [modèle (3)], compte tenu des valeurs observées pour alpha »relation (2)].

\begin{tabular}{lcccccc}
\hline & $\mathrm{Cd}_{\max }$ & $\mathrm{Cd}_{\min }$ & $\mathrm{Cd}_{\max } \mathrm{Cd}_{\min }$ & $\mathrm{Ch}_{\text {min }}$ & $\mathrm{Ch}_{\text {max }}$ & $\mathrm{Ch}_{\text {min }} \mathrm{Ch}_{\max }$ \\
\hline$k=0,1$ & 1,53 & 0,54 & 2,83 & 0,95 & 1,07 & 0,89 \\
$k=0,2$ & 1,46 & 0,57 & 2,56 & 0,91 & 1,15 & 0,79 \\
$k=0,3$ & 1,39 & 0,62 & 2,24 & 0,87 & 1,23 & 0,71 \\
$k=0,4$ & 1,33 & 0,66 & 2,02 & 0,83 & 1,32 & 0,63 \\
$k=0,5$ & 1,26 & 0,71 & 1,77 & 0,79 & 1,41 & 0,56 \\
\hline
\end{tabular}




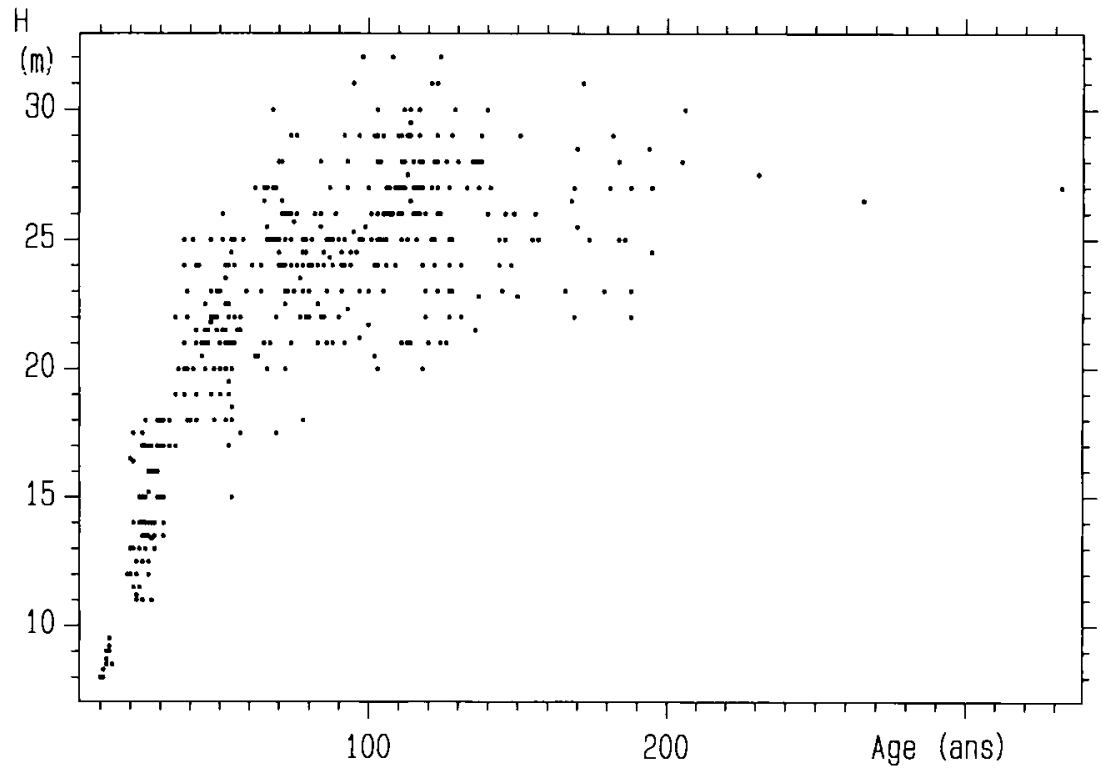

Fig 3. Hauteur $H$ en fonction de l'âge à 2,80 m pour 505 chênes pédonculés des forêts d'Amance et de Champenoux (54).

Ceci nous a amené à opter pour la valeur $k=$ 0,3 . Il vient alors :

$$
\begin{aligned}
& C d=\text { alpha } a^{0,7}=[(H / D) / f(A g \theta)]^{0,7} \\
& C h=\text { alpha }{ }^{0,3}=[(H / D) / f(A g \theta)]^{-0,3}
\end{aligned}
$$

En fait, nous avons également essayé les valeurs $k=0,2$ et $k=0,4$ dans les applications qui suivent. Ces essais ont montré d'une part, que la valeur $k=0,3$ semble effectivement la plus pertinente et d'autre part, que la précision du choix n'est pas critique, car les résultats obtenus avec les 2 autres valeurs sont relativement peu différents.

\section{PREMIËRES APPLICATIONS}

Les données dendrochronologiques et dendrométriques disponibles en forêts d'Amance et de Champenoux sur le chêne pédonculé (505 arbres) et sur le chêne sessile (529 arbres) ont été mises à profit pour une première utilisation pratique des 2 indices $C d$ et $C h$ proposés.

Les coefficients $a$ et $b$ de la relation (1) ont été établis pour le chêne sessile, selon la même démarche que pour le chêne pédonculé. On a dans ce cas :

$a=4,744 ; b=4,94 \cdot 10^{-3}$ (écart type $=2,50$ $\left..10^{-4}\right)$

écart type initial de $\log (H / D)=0,226$; écart type résiduel de $\log (H / D)=0,158$.

Pour chaque arbre ont été ensuite calculés les indices $C d$ et $C h$ à l'aide des formules (4) et (5).

\section{La croissance radiale à long terme. Utilisation de Cd}

L'ensemble des largeurs de cernes mesurées (environ 40400 pour le chêne pédon- 
culé et 45500 pour le chêne sessile) ont été standardisées, c'est-à-dire transformées en indices de croissance, exprimés en \%, dans lesquels l'effet de l'âge courant (âge de l'arbre au moment de la fabrication du cerne) a été éliminé. La technique de standardisation utilisée est exposée plus précisément par ailleurs (Becker, 1989). Elle consiste à faire le rapport de la largeur mesurée à une largeur de référence au même âge courant, préalablement établie et traduisant la loi biologique de vieillissement de l'espèce étudiée dans la région considérée.

Ces indices de croissance ont ensuite été multipliés par le coefficient correcteur de diamètre $C d$ de l'arbre correspondant. L'évolution dans le temps, depuis le début du $X I X^{\theta}$ siècle, de la moyenne générale des indices de croissance ainsi compensés apparaît dans les figures 4 (chêne pédonculé) et 5 (chêne sessile).

Alors que l'évolution des indices non compensés ne montrait pratiquement aucune tendance à long terme (Nieminen, 1988), on constate à présent une très nette dérive positive depuis 1830 , nettement plus forte d'ailleurs chez le chêne sessile (environ $+60 \%$ ) que chez le chêne pédonculé (environ $+32 \%$ ). La différence

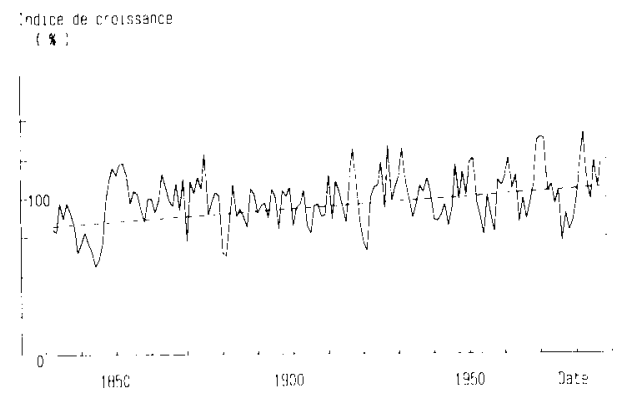

Fig 4. Évolution moyenne de l'indice de croissance radiale dégagée de l'effet de la compétition pour le chêne pédonculé en forêts d'Amance et de Champenoux (54).

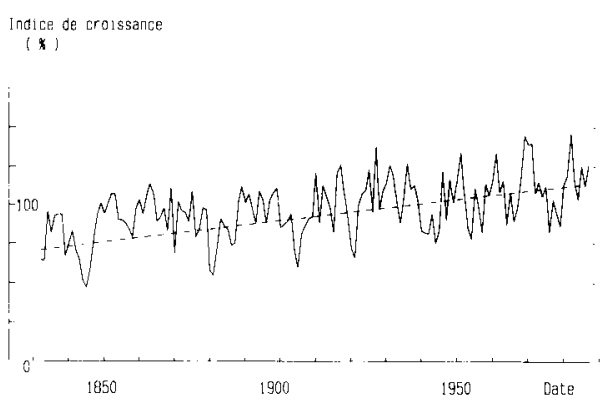

Fig 5. Évolution moyenne de l'indice de croissance radiale dégagée de l'effet de la compétition pour le chêne sessile en forêts d'Amance et de Champenoux (54).

de comportement des 2 espèces sera analysée et commentée par ailleurs. Toujours est-il que ce résultat apparaît plus cohérent avec les conclusions d'études récentes sur des essences diverses, résineuses et feuillues, tant en France (Becker, 1989; Bert et Becker, 1990; Picard, comm pers) que dans d'autres pays européens (Hari et al, 1984; Kenk et Spiecker, 1988; Hartmann et Schneider, 1989; Briffa, 1990). Simultanément, les tendances observées ici nous apparaissent comme un premier élément très encourageant pour une validation indirecte de la pertinence de l'indice correcteur de diamètre $C d$.

On pourrait s'étonner de ce résultat, obtenu avec un coefficient unique appliqué à tous les cernes d'un arbre, alors que le statut de compétition de ce dernier par rapport à ses voisins a pu varier au cours du temps. Une reconstitution plus fine est, bien sûr, pratiquement impossible, du moins sur un grand nombre d'individus, et $C d$ est une valeur moyenne intégrant toute la vie de l'arbre. Le caractère décisif de son apport réside dans le fait qu'il a pu en grande partie corriger, dans les données initiales, le problème de "confusion" (au 
sens statistique du terme) qui existe entre date, âge et statut de compétition : plus concrètement, le fait que les cernes les plus anciens correspondent majoritairement à des arbres de taillis-sous-futaie, et les cernes les plus récents à des arbres de futaie. Ainsi, bien que la productivité actuelle à l'échelle du peuplement soit plus importante qu'au siècle dernier, ceci est compensé en moyenne, à l'échelle de l'arbre, par une croissance radiale semblable, en réponse à une compétition plus importante due au traitement sylvicole de la futaie.

\section{Déterminisme écologique de la croissance en hauteur. Utilisation de Ch}

Pour chaque arbre de hauteur observée $H_{0}$, on calcule une hauteur compensée $H_{c}$ $\left(H_{c}=H_{o} \cdot C h\right)$ qui, si le coefficient $C h$ est pertinent, devrait pouvoir être comparée à celle de tout autre arbre indépendamment de leur statut respectif de compétition passée.

Auparavant, une autre opération très importante s'impose, destinée à tenir compte de l'âge actuel des arbres. Pour ce faire, nous avons calculé un indice de hauteur Ihauc $u_{c}$ rapport de la hauteur compensée $H_{c}$ à une hauteur de référence au même âge. Cette hauteur de référence $H_{\text {ref }}$ est celle obtenue par un ajustement curvilinéaire de l'ensemble des données disponibles. Dans le cas du chêne pédonculé rapporté ici (fig 6), le modèle qui s'est avéré le plus satisfaisant est :

$$
\log \left(H_{r e f}\right)=a+b \cdot A g e^{-0,5}+c \cdot \log (A g e)
$$

dans lequel

$$
a=5,77 ; b=-10,03 \text { (écart type }=0,54 \text { ); }
$$$$
c=-0,329 \text { (écart type }=0,039 \text { ) }
$$

écart type initial de $\log \left(H_{\text {ref }}\right)=0,293$; écart type résiduel de $\log \left(H_{\text {ref }}\right)=0,111$.

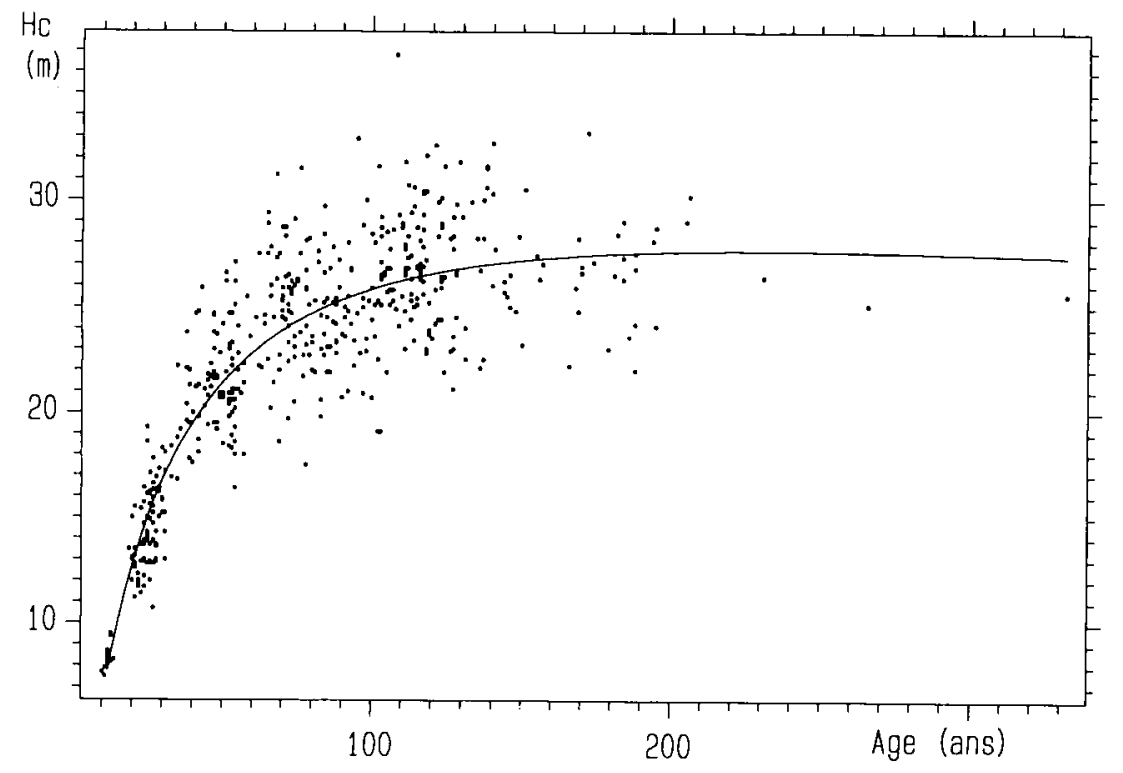

Fig 6. Hauteur compensée $H_{c}$ (dégagée de l'influence de la compétition passée) en fonction de l'âge à $2,80 \mathrm{~m}$ pour 505 chênes pédonculés des forêts d'Amance et de Champenoux (54), et ajustement. 
Remarque : les calculs ont été également faits en ne prenant en compte que les seuls arbres de futaie, dont les âges ne dépassent pas 130 ans; l'ajustement obtenu est pratiquement identique au précédent, ce qui, indirectement, contribue à accréditer la pertinence des coefficients $\mathrm{Ch}$ mis en œuvre.

Le même type de calcul a également été fait avec les hauteurs réelles mesurées $H_{0}$, pour obtenir des indices de hauteur Ihau $_{o}$, dégagés de l'influence de l'âge, mais pas de celle de la compétition passée.

Ce sont les 2 séries d'indices $\operatorname{lha}_{0}$ et thau $_{c}$ que nous avons cherché à "expliquer" en fonction des données stationnelles disponibles sur chaque placette d'étude. Cette comparaison porte sur les seules 76 placettes de la forêt d'Amance où le chêne pédonculé est bien représenté, après calcul des indices moyens par placette.

Le détail de cette étude sera exposé par ailleurs. Parmi les variables explicatives potentielles prises en compte dans un processus de régression multiple pas à pas, 4 se sont avérées participer significativement ( $F$ partiel supérieur à 4 ) à l'explication de l'indice de hauteur :

-2 indices floristiques $I t_{1}$ et $t_{2}$, obtenus a partir d'une analyse factorielle des correspondances sur les relevés de végétation de chaque placette; $I f_{1}$ est en rapport avec le niveau nutritionnel et $/ f_{2}$ avec la disponibilité de l'eau;

- la profondeur d'apparition d'un niveau d'argile lourde $p A l o$, qui doit limiter la profondeur d'enracinement;

- la profondeur d'apparition des premières traces d'hydromorphie temporaire $\mathrm{pHy}$; en fait, la liaison observée est apparue parabolique, ce qui est pris en compte par l'utilisation des variables $p H y$ et $p H y^{2}$ dans la régression.
Pour thau $u_{c}$, le taux global de variance expliquée est de $29 \%$. Pour $I_{h a u_{0}}$, il tombe à $22 \%$, et la contribution de $I f_{1}$ n'est plus significative. L'écart se creuse lorsque l'on ne s'intéresse qu'aux seules placettes à hydromorphie temporaire profonde $(\mathrm{pHy}$ $>35 \mathrm{~cm}$ ) : $54 \%$ de la variance est alors expliquée avec $I_{h a u_{c}}$ et $37 \%$ seulement avec Ihau.

L'intérêt du second indice de compétition $\mathrm{Ch}$ est donc également confirmé. La faiblesse relative des taux d'explication obtenus est commentée par ailleurs. Elle tient surtout à la difficulté d'une analyse quantitative pertinente des conditions édaphiques sur les substrats correspondants (limons sur marnes) et à la variabilité relativement faible des types de stations rencontrés.

\section{CONCLUSIONS ET PERSPECTIVES}

Nous sommes très conscient de ce que le caractère mi-déterministe, mi-empirique et intuitif de l'approche proposée peut avoir de déroutant, voire d'irritant. En fait, la présente étude a un caractère essentiellement exploratoire, et seule l'épreuve des exemples d'utilisation permettra de valider ou de rejeter définitivement cette approche, ou de conduire à des modifications dans la construction des indices de compétition $\mathrm{Cd}$ et $\mathrm{Ch}$. C'est ce que nous avons commencé à faire avec les données actuellement disponibles sur les chênaies du plateau lorrain. Les prochains tests devraient porter sur le sapin pectiné dans le massif du Jura et sur le hêtre commun dans l'étage montagnard des Vosges. En ce qui concerne la construction des indices, le modèle (3) en particulier devrait pouvoir être amélioré, en recherchant une formulation qui ne fasse varier que très peu $C d$ et $C h$ lorsqu'alpha est proche de 1. 
Le point le plus critique nous semble en fait résider dans le «postulat» de base avancé, à savoir que le facteur d'élancement d'un arbre dépend seulement de son âge et de la compétition moyenne qu'il a subie, et non des conditions de station. II s'agit beaucoup plus d'une "intuition forte" que d'une certitude, même si un certain degré de compatibilité avec la «loi de Eichhorn élargie" peut être mis en avant.

Quoi qu'il en soit, l'utilisation des indices $C d$ et $C h$, dans leur forme actuelle, impose un certain nombre de contraintes :

- l'âge de chaque arbre doit être connu avec une précision suffisante; cette précision doit être d'autant plus grande que l'arbre est plus jeune; ceci nécessite le plus souvent un carottage à cœur et un comptage des cernes, ce qui, pour diverses raisons pratiques, peut être un élément plus ou moins limitant;

- les coefficients $a$ et $b$ de la relation (1) liant $(H / D)$ à l'âge doivent être établis par essence, bien sûr, mais devraient sans doute l'être aussi par région naturelle (au sens de la typologie des stations); ceci tempère d'ailleurs pour une part importante le caractère abrupt du postulat évoqué, mais implique une étude spécifique préalable, sur quelques centaines d'arbres échantillonnant au mieux la gamme des âges et celle des statuts de compétition existants; ultérieurement, on pourra juger de la variabilité ou de la robustesse de l'estimateur du facteur d'élancement (H/D) en fonction de l'âge.

Si la pertinence des indices de compétition proposés venait à être confirmée, ceux-ci pourraient rendre de grands services:

- d'une part, dans l'étude des relations station-production (indice $\mathrm{Ch}$ ), en particulier dans les forêts où l'on sait que la sylviculture passée a été très perturbée mais sans pouvoir en quantifier les effets sur les hauteurs disponibles aujourd'hui;
- d'autre part, en dendroécologie (indice $C d)$, dans l'étude du déterminisme écophysiologique de la croissance radiale des grandes essences forestières.

\section{RÉFÉRENCES}

Assmann E (1955) Die Bedeutung der "erweiterten Eichhorn'schen Gesetzes" für die Konstruktion von Fichtenertragstafeln. Forstwiss Centralbl (Hamb), 321-330

Becker M (1978) Définition des stations en forêt de Haye. Potentialités du hêtre et du chêne. Rev For Fr 36, 93-124

Becker $M$ (1989) The role of climate on present and past vitality of Silver fir forests in the Vosges Mountains of Northeastern France. Can J For Res 19, 1110-1117

Becker M, Le Tacon F, Timbal J (1980) Les plateaux calcaires de Lorraine. Types de stations et potentialités forestières. ENGREF, Nancy, 268 p + tableau, ISBN 2-85710-004-3

Bert GD, Becker M (1990) Vitalité actuelle et passée du sapin (Abies alba Mill) dans le Jura. Etude dendroécologique. Ann Sci For 47, 395-412

Briffa KR (1990) Increasing productivity of "natural" growth of conifers in Europe over the last century. Int Symposium "Tree rings and Environment", 3-9 Sept 1990, Lund, Suède. Lundqua Rep (à paraître)

Day MW, Bey CF, Rudolph VJ (1960) Site index for planted Red pine by the 5-year growth intercept method. J For 58, 198-202

Décourt N (1973) Production primaire, production utile : méthodes d'évaluation, indices de productivité. Ann Sci For 30, 219-238

Décourt N, Le Tacon F (1970) L'épicéa commun (Picea excelsa) sur les plateaux calcaires de l'Est de la France. Essai de prévision de la production à l'aide de déterminations pédologiques simples. Ann Sci For 27, 255-286

Delpech R, Dumé G, Galmiche P (1985) Typologie des stations forestières. Vocabulaire. IDF, Paris, 243 p, ISBN 2-904740-05-8

Gehrhardt (1909) Über BestandesWachstumgesetze und ihre Anwendung zur Austellung von Ertragstafeln. Allg ForstJagdztg 85, 117-128 
Hari $P$, Arovaara $H$, Raunemaa $T$, Hautojärvi $A$ (1984) Forest growth and the effects of energy production: a method for detecting trends in the growth potential of trees. Can J For Res 14, 437-440

Hartmann DA, Schneider O (1990) Études régionales sur l'accroissement de l'arbre et du peuplement. In: Sanasilva Tagungsberichte. Tagung "Waldwachstum und Waldschäden", WSI, Birmensdorf, Suisse

Kenk G, Spiecker H (1988) Einige Ergebnisse zum aktuellen und früheren Wachstumsverhalten von Fichten. KfK-PEF 35, 371-381

Le Goff N (1984) Indice de productivité des taillis-sous-futaie de chêne dans la région Centre. Ann Sci For 41, 1-33

Le Tacon F, Millier C (1970) Influence des conditions de nutrition minérale sur la croissance de l'épicéa commun sur les plateaux calcaires de l'Est de la France. Ann Sci For 27, 335-353

Lévy $G$, Becker $M$ (1987) Le dépérissement du sapin dans les Vosges : róle primordial de déficits d'alimentation en eau. Ann Sci For 44, 403-416

Nieminen TM (1988) Étude dendroécologique du chêne (pédonculé et sessile) et du hêtre dans une forêt de la plaine lorraine (forêt domaniale d'Amance). DEA Biologie forestière, Univ Nancy 1, $58 \mathrm{p}$

Oswald $H$ (1969) Conditions forestières et potentialité de l'épicéa en Haute-Ardèche. Ann Sci For 26, 183-224

Oswald H (1984) Production ef sylviculture du Douglas en plantations. Rev For Fr 36, 268278

Pardé J, Bouchon J (1988) Dendrométrie. $2^{\theta}$ édn, ENGREF, Nancy, $328 \mathrm{p}$ 